\title{
Adipose-derived mesenchymal stem cells from obese mice prevent body weight gain and hyperglycemia
}

Yicheng $\mathrm{Qi}^{1+}$, Wen Liu ${ }^{1 \dagger}$, Xiangsheng Wang ${ }^{2}$, Nan Lư ${ }^{1}$, Minglan Yang ${ }^{1}$, Wei Liư ${ }^{2}$, Jing Ma', Wei Liu', Wenjie Zhang ${ }^{2^{*}}$ and Shengxian $\mathrm{Li}^{\mathrm{i}^{*}}$

\begin{abstract}
Changes that occur to the stem cell microenvironment with disease are a major consideration that may affect the behavior and potential therapeutic efficacy of mesenchymal stem cells (MSCs). The purpose of this study is to evaluate the effects of adipose-derived MSCs (ADSCS) from obese mice with hyperglycemia on body weight and glucose homeostasis. After 10 weeks of high-fat diet, mice were injected with phosphate-buffered saline (PBS) and ADSCs derived from normal mice (N-ADSCs) or obese mice (O-ADSCs), respectively. Mice fed with standard rodent chow were injected with PBS and served as normal controls. Obese mice treated with O-ADSCs showed less body weight gain than those receiving PBS or N-ADSCs. The mice that received ADSCs, especially O-ADSCs, also showed improvement in obesity-related hyperglycemia. In particular, the inguinal fat was reduced in obese mice receiving O-ADSCs compared with other groups, probably caused by the increased lipolysis of inguinal fat. Moreover, ADSC infusion restored insulin receptor (INSR) expression in the muscle of obese mice. Differential expression of the CD90 surface marker was slightly increased, while monocyte chemoattractant protein 1 (MCP-1) was reduced in O-ADSCS compared to N-ADSCs. These data provide a theoretical basis that autologous ADSCs from obese individuals may be more effective for treating obesity and related hyperglycemia.
\end{abstract}

Keywords: Adipose-derived MSCs, Lipolysis, INSR, Obesity, Hyperglycemia

\section{Introduction}

At present, there is a worldwide epidemic of obesity, which often leads to the development of type 2 diabetes mellitus (T2DM) and its associated medical and economic challenges. Over the last four decades, insulin resistance (IR), caused by obesity, has been identified as a major trigger of the T2DM epidemic in China [1]. To

\footnotetext{
*Correspondence: wenjieboshi@aliyun.com; rj_shengxianli@163.com

${ }^{\dagger}$ Yicheng Qi and Wen Liu contributed equally to this work.

${ }^{2}$ Department of Plastic and Reconstructive Surgery, Shanghai 9th People's Hospital, School of Medicine, Shanghai Jiaotong University, Shanghai Key Laboratory of Tissue Engineering, No. 639 Zhizaoju Road, Huangpu Area, Shanghai 200011, China

'Department of Endocrinology and Metabolism, Renji Hospital, School of Medicine, Shanghai Jiaotong University, No. 160 Pujian Road, Pudong New Area, Shanghai 200127, China
}

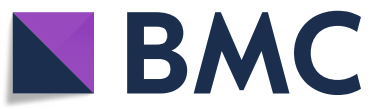

(c) The Author(s). 2021 Open Access This article is licensed under a Creative Commons Attribution 4.0 International License, which permits use, sharing, adaptation, distribution and reproduction in any medium or format, as long as you give appropriate credit to the original author(s) and the source, provide a link to the Creative Commons licence, and indicate if changes were made. The images or other third party material in this article are included in the article's Creative Commons licence, unless indicated otherwise in a credit line to the material. If material is not included in the article's Creative Commons licence and your intended use is not permitted by statutory regulation or exceeds the permitted use, you will need to obtain permission directly from the copyright holder. To view a copy of this licence, visit http://creativecommons.org/licenses/by/4.0/. The Creative Commons Public Domain Dedication waiver (http://creativecommons.org/publicdomain/zero/1.0/) applies to the data made available in this article, unless otherwise stated in a credit line to the data.

date, effective therapies for improving insulin resistance and curbing the development of obesity-related hyperglycemia have yet to be developed.

Mesenchymal stem cells (MSCs), which are characterized by their ability to self-renew and multipotentiality, have been identified in a number of tissues including bone marrow, fetal annexes, adipose, dental, and liver tissues [2]. The minimal characterization criteria for MSCs, as proposed by the International Society for Cellular Therapy in 2006, are as follows: (1) plasticadherent; (2) expression of CD73, CD90, and CD105 and lack expression of CD45, CD34, CD14, CD11b, CD79 $\alpha, C D 19$, and HLA-DR surface markers; and (3) multilineage differentiation potential toward adipocytes, osteocytes, and chondrocytes [3]. Of the many types of 
MSCs available, adipose-derived MSCs (ADSCs) are considered to have a number of advantages, including few ethical concerns (versus cells from fetal/neonatal tissues), ease of accessibility, and an abundance of cells from multiple sources [4]. Besides, previous evidences also confirmed the anti-obesity and anti-hyperglycemia benefits of ADSCs. Cao et al. revealed that ADSCs from healthy C57BL/6 mice reduced body weight and blood glucose levels in high-fat diet-induced obese mice. Further, the ADSC-injected mice displayed lower levels of macrophage (F4/80+) infiltration, interleukin-6 (IL-6), and nucleotide-binding oligomerization domain 2 (NOD2) in liver tissue, resulting in improved insulin resistance. Decreased macrophage infiltration was also discovered in pancreases after ADSC infusion, which may partially contribute to the protection of pancreatic $\beta$-cell mass [5]. In another report, it was found that the infusion of ADSCs alleviated hyperglycemia and insulin resistance in T2DM rats via restoration of glucose transporter-4 (GLUT4) and INSR expression on the cell membrane of the skeletal muscle, liver, and adipose tissue [6]. Others have shown that the infusion of ADSCs also has rapidly improved blood glucose levels (within $24 \mathrm{~h}$ ) in T2DM rats which involved changes in the regulation of glycogen metabolism and gluconeogenesis via the AMPK signaling pathway [7]. Although all of these studies strongly suggest the utility of ADSCs in clinical applications, especially with regard to obesity and T2DM, the selection of allogeneic or autologous MSCs is still in question.

The allogeneic MSCs derived from donators are "offthe-shelf" cellular therapy. Although MSCs are previously identified as immune-privileged, it is also reported that allogeneic MSCs trigger local inflammation by allospecific T-effector cells. Side effects are even accelerated after multiple infusions of allogeneic MSCs by boosting memory allo-response, which limits their further clinical application [8]. Fortunately, these problems were evitable in autologous MSC therapy. Autologous MSC therapy has higher acceptance and lower risk of infectious diseases. However, disease status always affects the function of MSCs. Obese adipose tissue is characterized by chronic inflammatory state, hypoxia, and metabolic disturbance [9]. These disorders may lead to impaired functions of ADSCs including multipotent differentiation ability, metabolism, and immunomodulation, which presumably reduce the curative effect of autologous ADSCs [10-12]. Thus, we wonder whether ADSCs isolated from normal control and obese mice showed different efficacy.

In the present study, we used a high-fat diet-induced mouse model of obesity and then compared the therapeutic efficacy of ADSCs derived from normal and obese donors on body weight and glucose homeostasis in obese mouse recipients. By taking this approach, we hope to better understand the underlying mechanisms affecting autologous ADSCs and their potential for use in T2DM therapy.

\section{Materials and methods \\ Animals and experimental design}

Since C57BL/6 mice are predisposed to high-fat dietinduced metabolic syndrome, we purchased 4-week-old male C57BL/6 mice from the Shanghai Jihui Laboratory of Animal Care for use in this study. After a 1-week period of adaptation to the environment, mice were fed a standard chow diet (10\% of calories from fat) or a high-fat diet (HFD; $60 \%$ of calories from fat). Mice fed with the standard chow diet were used as normal controls. After 10 weeks, the HFD mice were randomized into 3 groups. Each group received a tail vein injection on days 0,4 , and 9. Group 1 received phosphatebuffered saline (PBS) $(n=5)$; group 2 received $5 \times 10^{5}$ ADSCs from normal control mice (N-ADSCs) $(n=5)$, and group 3 received $5 \times 10^{5}$ ADSCs from obese mice (O-ADSCs) ( $n=6)$. Body weight and blood glucose levels were continuously monitored and percent change calculated. Two months after tail vein infusion, intraperitoneal glucose tolerance (IPGTT) and insulin releasing test (IRT) were performed after a 12-h fast. At the end of the experiment, mice were anesthetized with $10 \%$ chloral hydrate, and dissected tissues were frozen in liquid nitrogen immediately after sacrifice. Inguinal, epididymal fat pads were weighed after excision.

\section{ADSC isolation and culture}

Fresh inguinal fat was isolated from normal control and obese mice and washed with Dulbecco's phosphatebuffered saline (D-PBS, Gibco). Adipose tissues were cut into small pieces and then digested with $2 \mathrm{mg} / \mathrm{mL}$ type I collagenase (Sigma-Aldrich) for $30 \mathrm{~min}$ at $37^{\circ} \mathrm{C}$ on a shaker. Cells were resuspended after centrifugation at $1500 \mathrm{rpm}$ for $5 \mathrm{~min}$ and then filtered through a $40-\mu \mathrm{m}$ nylon filter mesh (BD Falcon) to remove any tissue residue. After washing twice, cells were resuspended in alpha-MEM medium (Gibco) containing 10\% fetal bovine serum (Gibco) and seeded in T75 tissue culture flasks (BD Falcon). Cells were cultured in a humidified $5 \% \mathrm{CO}_{2}$ incubator at $37^{\circ} \mathrm{C}$. Floating cells were removed after $24 \mathrm{~h}$ and the media changed after 3 days. At $90 \%$ confluence, cells were detached with $0.25 \%$ trypsinEDTA and passaged.

\section{Intraperitoneal glucose tolerance and insulin releasing test}

For IPGTT, all mice received an intraperitoneal injection of glucose $(2 \mathrm{~g} / \mathrm{kg})$ after an overnight fast. At $0,15,30$, 60 , 90, and 120 min after glucose load, blood glucose 
levels were measured with a glucometer (Roche). At 0, 15 , and $30 \mathrm{~min}, 0.1-\mathrm{mL}$ blood samples were collected from the orbital venous plexus. Immediately after centrifugation, plasma was obtained and promptly stored at $-80^{\circ} \mathrm{C}$. Insulin assay was subsequently performed by ELISA (Alpco) according to the manufacturer's protocol. HOMA-IR index was calculated by the equation: HOMA-IR index $=($ FBG $[$ in $\mathrm{mmol} / \mathrm{L}] \times$ FINS [in units $/$ L])/22.5.

\section{RNA extraction and quantitative RT-PCR}

Total RNA was extracted from liver, muscle, and adipose tissues using the RNeasy Kit (Qiagen), followed by converting into the first-strand $\mathrm{CDNA}$ with the cDNA synthesis kit (Takara). Quantitative RT-PCR was performed using SYBR Master Mix (Takara) and a LightCycler 480 System (Roche). The quantity of mRNA was normalized to $\beta$-actin in the liver and muscle and to $36 \mathrm{~B} 4$ in adipose, respectively. The primer sequences used for this study are provided in Supplement Table 1.

\section{Western blot analysis}

Total protein was extracted from liver, muscle, and adipose tissues for Western blot analysis using a standard protocol. Thirty micrograms of protein was separated by SDS-PAGE and then transferred to a PVDF membrane (Millipore Corp). After blocking, the membranes were incubated overnight at $4^{\circ} \mathrm{C}$ with primary antibodies to Scd-1 (1:2000), Adr- $\beta 3$ (1:2000), INSR (1:2000), and GAPDH (1:2000) (Abcam). Next, these membranes were incubated with horseradish peroxidase-conjugated secondary antibody (Cell Signaling Technology). Protein bands were illuminated using ECL Prime Western Blotting Detection Reagent (GE Healthcare). This assay was repeated at least three times and a representative Western blot was chosen for the figures.

\section{Flow cytometry}

Cell suspensions containing $1 \times 10^{6}$ cells were incubated in $100 \mu \mathrm{L}$ of diluted FITC-conjugated rat anti-mouse CD44, CD90, CD105, and CD45 antibodies (BD Biosciences) for $30 \mathrm{~min}$ at $4^{\circ} \mathrm{C}$. After surface staining, cells were washed and analyzed by flow cytometry (BD Biosciences). The appropriate isotype antibodies were used as controls.

\section{Statistical analyses}

Statistical analysis was performed using SPSS version 25 and Graph Pad Prism 8. Data were expressed as mean \pm SD. Student's $t$ test or ANOVA was performed to analyze the differences among groups. The area under the curve (AUC) of the IPGTT and IRT were calculated. $p<0.05$ was considered statistically significant.

\section{Results}

\section{Generation of obese mice}

The obese mouse model was established by feeding the mice with HFD. Compared to the normal control group fed standard chow, body weight in the animals receiving the HFD increased significantly after 10 weeks (Fig. 1a, $26.72 \pm 0.67 \mathrm{~g}$ vs. $33.73 \pm 2.02 \mathrm{~g}, p<0.001)$. In addition, fasting blood glucose (FBG) levels in the obese mice were significantly higher than in the normal controls (Fig. 1b, $8.72 \pm 1.15 \mathrm{mmol} / \mathrm{L}$ vs. $6.75 \pm 0.82 \mathrm{mmol} / \mathrm{L}, p<0.001)$. Further, obese mice had impaired glucose tolerance in the IPGTT. HFD also induced insulin resistance with higher insulin levels (Fig. 1c). These results indicate that our obese mouse model is appropriate for use in our study.

\section{ADSC infusion reduced body weight}

To explore the effect of ADSCs on body weight, we injected ADSCs into obese mice via the tail vein. Interestingly, we found that injection of O-ADSCs significantly slowed the increase in weight of mice compared to those injected with PBS or N-ADSCs (Fig. 2a). We next measured the weights of epididymal fat and inguinal fat at the end of the observation period and found that infusion of ADSCs failed to influence the weight of epididymal fat pads. However, inguinal fat pad weight was significantly reduced in mice injected with $\mathrm{O}$ ADSCs as compared to mice injected with N-ADSCs or PBS (Fig. 2b). We next evaluated the expression of genes associated with adipogenesis and lipolysis in adipose tissues to determine the probable cause of the observed reduction in weight. As shown in Fig. 2c-e, obese mice injected with both $\mathrm{N}$ - and O-ADSCs were observed to downregulate stearoyl-CoA desaturase 1 (Scd-1) at both the transcript and protein levels in epididymal fat, although mice injected with O-ADSCs showed the most dramatic decline. Meanwhile, O-ADSCs restored the $\beta 3$ adrenergic receptor (Adr- $\beta 3$ ) expression in inguinal fat of obese mice (Fig. $2 \mathrm{f}-\mathrm{h}$ ). These data suggest that ADSCs, especially O-ADSCs, have a protective effect in obesity by partially increasing lipolysis in inguinal adipose tissue.

\section{Infusion of ADSCs improved glucose homeostasis and insulin sensitivity}

It has been indicated that obesity has a high risk for insulin sensitivity, we next examined whether infusion of ADSCs has an impact on glucose homeostasis. Obese mice receiving N-ADSCs or O-ADSCs had lower blood glucose levels than the controls, but the effect of treatment with O-ADSCs was more dramatic by day 2 after the first injection (Fig. 3a).

After 2 months of successive HFD feeding, the glucose tolerance became even more impaired in the obese mice (Fig. 3b). However, the infusion of ADSCs attenuated 


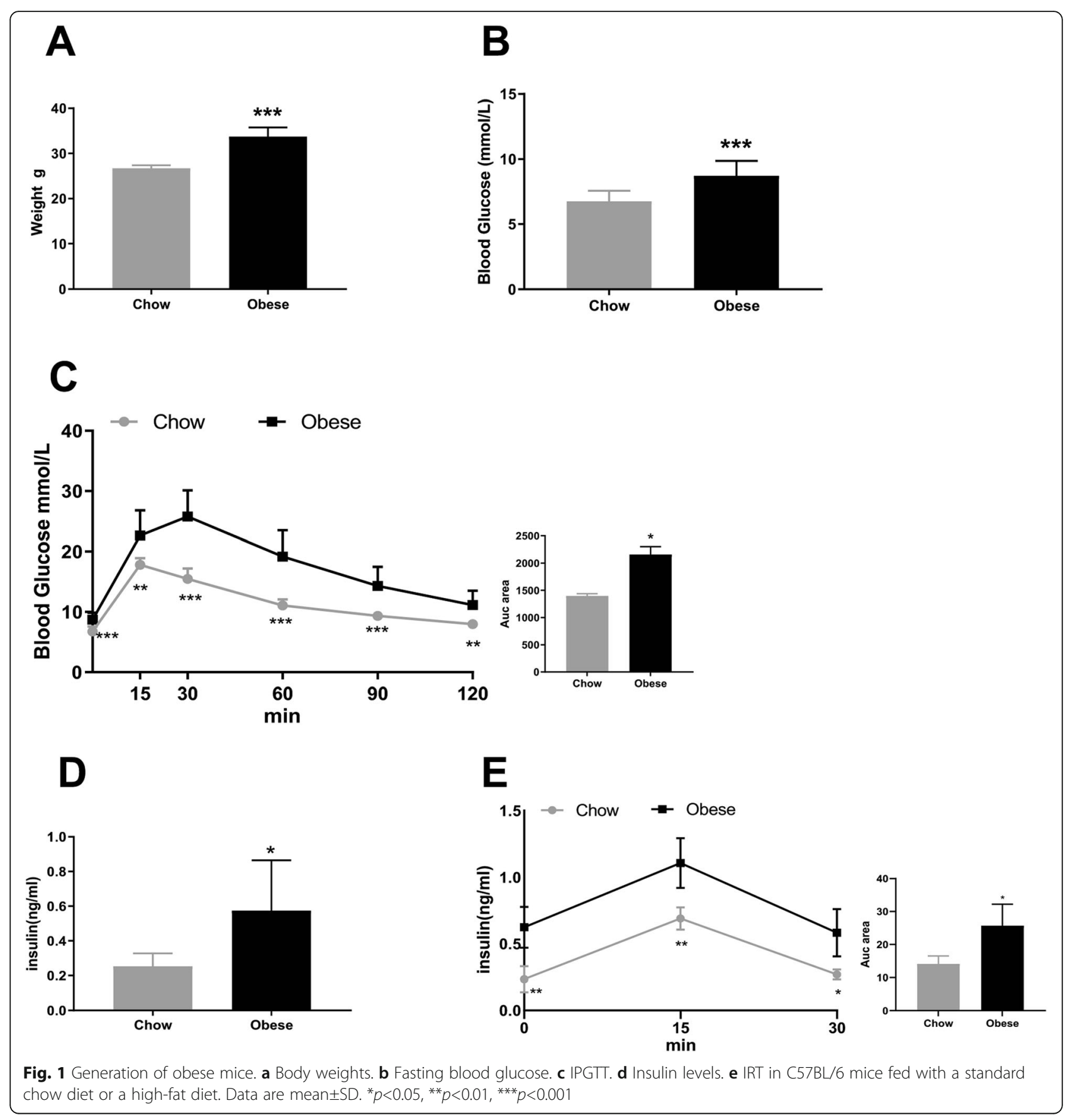

the decline in glucose tolerance, especially at the 15-min time point after glucose loading in the O-ADSCs group (Fig. 3c, d). Further, insulin response after glucose load was declined in the PBS group, but remained normal in both ADSC-treated groups (Fig. 3e). Obese mice receiving ADSCs also tended to display improved insulin sensitivity, but showed no statistically significant difference between the PBS and ADSCs groups (Fig. 3f).

To understand the underlying mechanisms, we measured the gene expression of insulin downstream molecules in adipose, liver, and muscle tissues (Fig. 3g-l). We found that the mRNA expression and protein levels of INSR in the muscle were dramatically elevated in obese mice receiving infusions of $\mathrm{N}$-ADSCs and O-ADSCs, compared to those treated with PBS, but mice receiving $\mathrm{O}$-ADSCs showed higher levels. In addition, INSR protein levels were increased in the liver after treatment with ADSCs. Taken together, these results demonstrated that ADSCs, especially O-ADSCs, improve glucose homeostasis and insulin sensitivity in obese mice. 


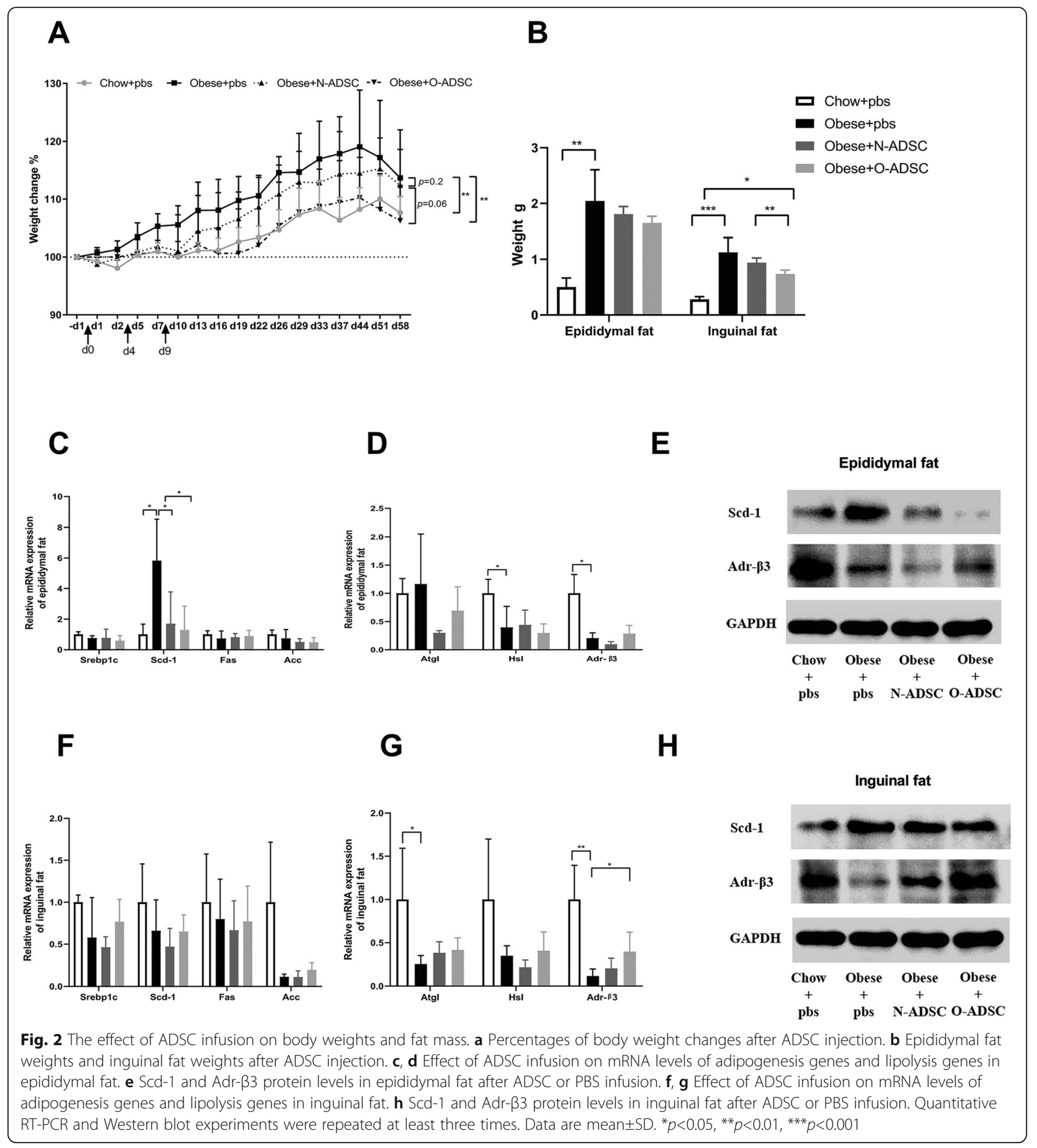

\section{Discussion}

This study revealed, for the first time, that O-ADSCs are a more effective cell-based therapy for treating obesity and deranged glucose homeostasis in HFD mice than NADSCs. Further, an improvement in body weight was accompanied by an increase in lipolytic gene expression in inguinal fat tissue after treatment with O-ADSCs. Moreover, O-ADSC treatment also tended to attenuate insulin resistance which coincided with the restoration of INSR expression in the muscle.

In this study, ADSCs were isolated from inguinal fat, a type of subcutaneous adipose tissue (SAT), which is less influenced by obesity and T2DM than visceral adipose tissue (VAT) [13]. In previous studies, the effect of ADSC treatment on body weight has been shown to be associated with disease progression. ADSCs reduce body 


\section{A}

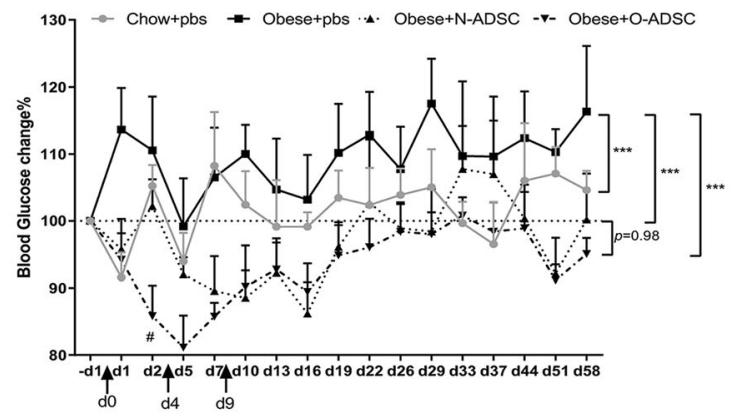

B

C

D

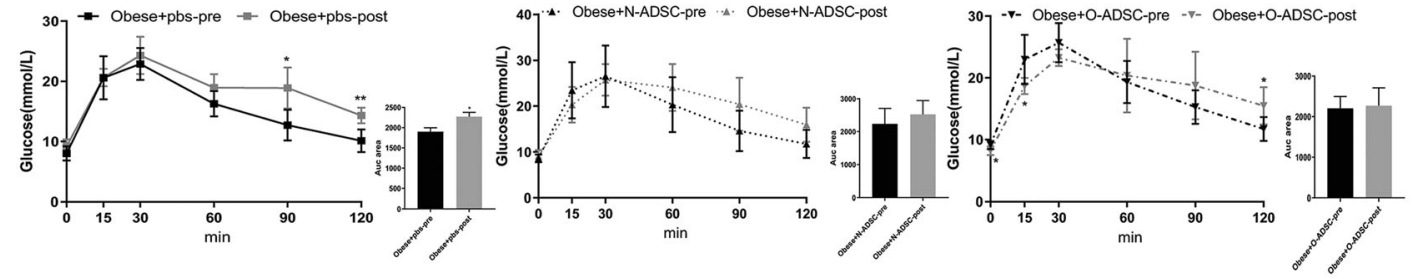

E

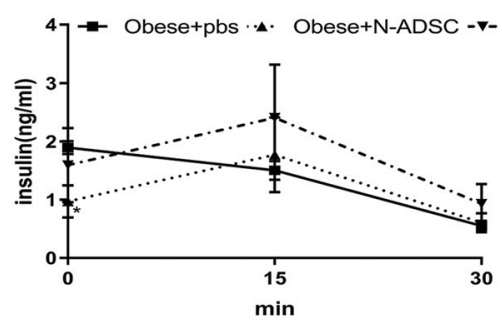

G

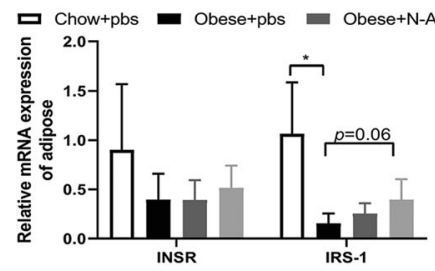

J

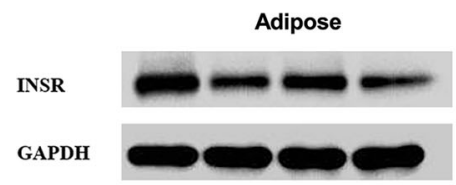

$\begin{array}{cccc}\text { Chow } & \text { Obese } & \text { Obese } & \text { Obese } \\ + & + & + & +\end{array}$

$\begin{array}{cccc}+ & + & + & + \\ \text { pbs } & \text { pbs } & \text { N-ADSC } & \text { O-ADSC }\end{array}$
F

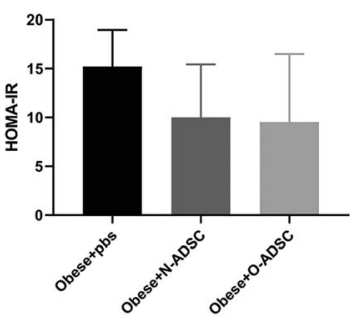

H

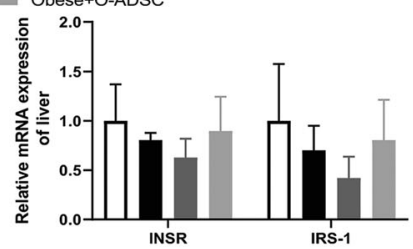

K

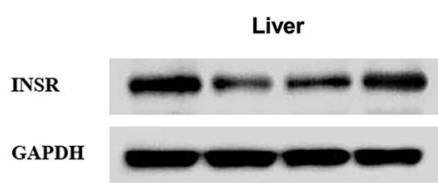

Chow Obese Obese Obese

$\begin{array}{cccc}+ & + & + & + \\ \text { pbs } & \text { pbs } & \text { N-ADSC } & \text { O-ADSC }\end{array}$

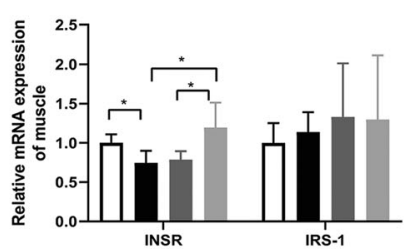

L

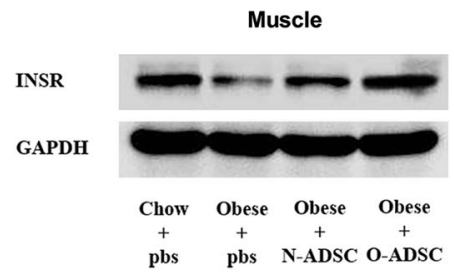

Fig. 3 (See legend on next page.) 
(See figure on previous page.)

Fig. 3 The effect of ADSC infusion on blood glucose levels and insulin sensitivity. a Percentages of blood glucose changes after ADSC injection. b-d IPGTT and area under the curve before and after PBS, N-ADSC, or O-ADSC injection. e Insulin releasing test before and after PBS, N-ADSC, or O-ADSC injection. $\mathbf{f}$ HOMA-IR after ADSC injection. $\mathbf{g - i}$ Effect of ADSC infusion on the mRNA expression of INSR and IRS-1 in insulin target tissues. j-I Effect of ADSC infusion on the protein levels of INSR in insulin target tissues. Quantitative RT-PCR and Western blot experiments were repeated at least three times. Data are mean \pm SD. ${ }^{*} p<0.05,{ }^{* *} p<0.01,{ }^{* * *} p<0.001$

weight in obese mice fed a HFD for 20 weeks with a negligible effect on T2DM, while body weight gradually increases during the later phase of diabetes $[5,6,14]$. In our study, we infused N-ADSCs into obese mice, as an early intervention, after 10 weeks of HFD feeding with no effect on body weight. In contrast, the infusion of OADSCs retarded body weight gain compared to the PBS and N-ADSCs groups, although a number of previous studies suggest that detrimental changes to the microenvironment in obese tissue impair the function of ADSCs [10]. It has been shown that MSCs are often quiescent and that their immunosuppressive properties are induced by inflammatory cytokines such as interferon- $\gamma$ (IFNY), interleukin-17 (IL-17), and tumor necrosis factor (TNF) in the microenvironment [15]. In addition, hypoxia may also enhance the function of MSCs via increased expression of IL-6, vascular endothelial growth factor (VEGF), and chemokines [16]. The data we report here and that of previous studies suggest that changes in the microenvironment might activate MSC function. Thus, it is important to consider factors, such as the location where ADSCs are obtained, the time between isolation and infusion, and cell activity/function, before MSC therapy.

Obesity is characterized by excessive fat accumulation [17]. Consistent with the previous report [5], we found no differences in the weights of the liver (data not show) and epididymal fat among control, N-ADSCs, and OADSCs groups in our study. Interestingly, we found that infusion of O-ADSCs decreased inguinal fat pad weight compared to treatment with PBS or N-ADSCs. Another study revealed that the anti-obesity effect of ADSCs was due to decreased lipogenesis and increased lipolysis through hormone-sensitive lipase activation and acetylCoA carboxylase1 suppression [18]. In the present study, we found that both N-ADSC and O-ADSC infusion inhibited lipogenesis by reducing Scd-1 expression of epididymal adipose tissue, while only O-ADSC treatment activated lipolysis by enhancing Adr- $\beta 3$ expression in inguinal adipose tissue.

Weight loss in obese and overweight patients with T2DM is associated with improvement in hyperglycemia [19]. As with previous research, our data suggest that ADSCs also significantly improve blood glucose levels in obese mice, especially in the O-ADSCs group. Animal studies have demonstrated that infusion of ADSCs reduces blood glucose levels via multiple mechanisms, including promotion of insulin production, improvement of insulin resistance, and regulation of hepatic glucose metabolism [20]. We found that ADSCs prevented the decline in insulin response to glucose load in HFD mice, which was related to the restoration of INSR expression in the muscle, especially seen in HFD mice treated with $\mathrm{O}$-ADSC infusion. The muscle is considered as a major target organ of insulin action largely involved in systemic insulin resistance. Considering that glucose uptake of the muscle accounts for approximately $80 \%$ of insulinmediated glucose utilization, the muscle plays a key role in whole-body glucose homeostasis [21]. Therefore, the restoration of INSR in the muscle may partially explain the relative advantage of O-ADSCs over $\mathrm{N}$-ADSCs in glycemic control.

We further characterized the properties of the two types of ADSCs at the cytological level. As supplement Fig. 1 shown, both N-ADSCs and O-ADSCs were fibroblast-like and normal growth. Both ADSCs displayed similar phenotypes with CD44, CD105, and CD45 expression, except that O-ADSCs expressed a slightly higher level of CD90 as compared to that of the $\mathrm{N}$-ADSCs. In addition, there was a downregulation of monocyte chemoattractant protein 1 (MCP-1) and interleukin-1 $\beta$ (IL-1 $\beta$ ) in O-ADSCs as compared with $\mathrm{N}$ ADSCs, which may partially explain the different effects of the two cell types. However, these data are not sufficient to identify the whole underlying mechanism. Further studies need to be conducted to clarify the cause of the phenomenon we have observed.

In conclusion, our results, for the first time, have provided evidence that O-ADSCs better reduced body weight and blood glucose levels than N-ADSCs. Further, the mechanism may be that O-ADSCs regulated the lipid metabolism of adipose tissue and improved insulin resistance of the skeletal muscle. The analysis of surface markers showed that O-ADSCs expressed slightly higher levels of CD90 while lower levels of MCP-1 than NADSCs. These findings demonstrate that O-ADSC infusion may be ideally used for controlling obesity and its related hyperglycemia and further support the development of autologous ADSC-based therapy for an obesityrelated T2DM.

\section{Abbreviations}

MSCs: Mesenchymal stem cells; ADSCs: Adipose-derived MSCs; PBS: Phosphate-buffered saline; N-ADSCs: ADSCs from normal control mice: O-ADSCs: ADSCs from obese mice; T2DM: Type 2 diabetes mellitus; IR: Insulin 
resistance; INSR: Insulin receptor; IRS-1: Insulin receptor substrate 1; MCP1: Monocyte chemoattractant protein 1; IL-6: Interleukin-6; NOD2: Nucleotidebinding oligomerization domain 2; GLUT4: Glucose transporter-4; HFD: Highfat diet; IPGTT: Intraperitoneal glucose tolerance; IRT: Insulin releasing test; DPBS: Dulbecco's phosphate-buffered saline; AUC: Area under the curve; FBG: Fasting blood glucose; Scd-1: Adipogenesis gene stearoyl-CoA desaturase 1; Adr- $\beta 3$ : Beta3-adrenergic receptor; SAT: Subcutaneous adipose tissue; VAT: Visceral adipose tissue; IFNY: Interferon-y; IL-17: Interleukin-17; TNF: Tumor necrosis factor; VEGF: Vascular endothelial growth factor; LL$1 \beta$ : Interleukin-1 $\beta$

\section{Supplementary Information}

The online version contains supplementary material available at https://doi. org/10.1186/s13287-021-02357-y.

Additional file 1: Supplement Table 1. Primer sequences used in quantitative RT-PCR.

Additional file 2: Supplement Figure 1. Both N-ADSCs and O-ADSCS were fibroblast-like and normal growth.

\section{Acknowledgements}

The authors thank Drs. Xiao-Dong Chen and David D. Dean for providing comments on the manuscript and editing of the text.

\section{Authors' contributions}

$S L, W Z, L W$, and $L W$ drafted the study design. YQ, WL, XW, and NL conducted the experiments and collected the data. JM and MY provided technical assistance. YQ wrote the first draft of the manuscript. SL and WZ were responsible for the critical revision of the manuscript. All authors read and approved the final manuscript.

\section{Funding}

This study was supported by the grants from the National Natural Science Foundation of China (No. 81800693, 82000814, 81800747, and 81800779), the Major Chronic Non-communicable Disease Prevention and Control Research, and National Key R\&D Program of China (2016YFC1305600 and 2016YFC1305602) and sponsored by the Shanghai Yangfan Program (19YF1428500).

\section{Availability of data and materials}

The datasets used and/or analyzed during the current study are available from the corresponding authors on reasonable request.

\section{Declarations}

\section{Ethics approval and consent to participate}

All experiments were performed according to standard protocols, in compliance with the Guide of the Animal Ethics Committee of Shanghai 9th People's Hospital, Shanghai Jiao Tong University School of Medicine (The reference number is $\mathrm{SH} 9 \mathrm{H}-2019-\mathrm{A} 700-1)$.

\section{Consent for publication}

Not applicable

\section{Competing interests}

The authors declare that they have no competing interests.

Received: 27 November 2020 Accepted: 26 April 2021

Published online: 06 May 2021

\section{References}

1. Wang T, Lu J, Shi L, Chen G, Xu M, Xu Y, et al. Association of insulin resistance and $\beta$-cell dysfunction with incident diabetes among adults in China: a nationwide, population-based, prospective cohort study. Lancet Diabetes Endocrinol. 2020;8(2):115-24. https://doi.org/10.1016/S2213-8587(1 9)30425-5.

2. Stagg J, Galipeau J. Mechanisms of immune modulation by mesenchymal stromal cells and clinical translation. Curr Mol Med. 2013;13(5):856-67. https://doi.org/10.2174/1566524011313050016.
3. Dominici M, Le Blanc K, Mueller I, Slaper-Cortenbach I, Marini F, Krause D, et al. Minimal criteria for defining multipotent mesenchymal stromal cells. The International Society for Cellular Therapy position statement. Cytotherapy. 2006;8(4):315-7. https://doi.org/10.1080/14653240600855905.

4. Tobita M, Orbay H, Mizuno H. Adipose-derived stem cells: current findings and future perspectives. Discov Med. 2011;11(57):160-70.

5. Cao M, Pan Q, Dong H, Yuan X, Li Y, Sun Z, et al: Adipose-derived mesenchymal stem cells improve glucose homeostasis in high-fat dietinduced obese mice. Stem Cell Res Ther 2015;6(208). https://doi.org/10.11 86/s13287-015-0201-3.

6. Hu J, Fu Z, Chen Y, Tang N, Wang L, Wang F, et al. Effects of autologous adipose-derived stem cell infusion on type 2 diabetic rats. Endocr J. 2015; 62(4):339-52. https://doi.org/10.1507/endocrj.EJ14-0584.

7. Xie M, Hao HJ, Cheng Y, Xie ZY, Yin YQ, Zhang Q, et al. Adipose-derived mesenchymal stem cells ameliorate hyperglycemia through regulating hepatic glucose metabolism in type 2 diabetic rats. Biochem Biophys Res Commun. 2017:483(1):435-41. https://doi.org/10.1016/j.bbrc.2016.12.125.

8. Consentius $C$, Reinke P, Volk HD. Immunogenicity of allogeneic mesenchymal stromal cells: what has been seen in vitro and in vivo? Regen Med. 2015;10(3):305-15. https://doi.org/10.2217/rme.15.14.

9. Blüher M. Adipose tissue dysfunction in obesity. Exp Clin Endocrinol Diabetes. 2009:117(6):241-50. https://doi.org/10.1055/s-0029-1192044.

10. Louwen F, Ritter A, Kreis NN, Yuan J. Insight into the development of obesity: functional alterations of adipose-derived mesenchymal stem cells. Obes Rev. 2018;19(7):888-904. https://doi.org/10.1111/obr.12679.

11. Ritter A, Friemel A, Kreis NN, Hoock SC, Roth S, Kielland-Kaisen U, et al. Primary cilia are dysfunctional in obese adipose-derived mesenchymal stem cells. Stem Cell Reports. 2018;10(2):583-99. https://doi.org/10.1016/j.stemcr.2 017.12.022.

12. Badimon L, Cubedo J. Adipose tissue depots and inflammation: effects on plasticity and resident mesenchymal stem cell function. Cardiovasc Res. 2017;113(9):1064-73. https://doi.org/10.1093/cvr/cvx096.

13. Serena C, Keiran N, Ceperuelo-Mallafre V, Ejarque M, Fradera R, Roche K, et al. Obesity and type 2 diabetes alters the immune properties of human adipose derived stem cells. Stem Cells. 2016;34(10):2559-73. https://doi. org/10.1002/stem.2429.

14. Wang M, Song L, Strange C, Dong X, Wang H. Therapeutic effects of adipose stem cells from diabetic mice for the treatment of type 2 diabetes. Mol Ther. 2018;26(8):1921-30. https://doi.org/10.1016/j.ymthe.2018.06.013.

15. Han X, Yang Q, Lin L, Xu C, Zheng C, Chen X, et al. Interleukin-17 enhances immunosuppression by mesenchymal stem cells. Cell Death Differ. 2014; 21(11):1758-68. https://doi.org/10.1038/cdd.2014.85.

16. Hung SC, Pochampally RR, Chen SC, Hsu SC, Prockop DJ. Angiogenic effects of human multipotent stromal cell conditioned medium activate the PI3KAkt pathway in hypoxic endothelial cells to inhibit apoptosis, increase survival, and stimulate angiogenesis. Stem Cells. 2007;25(9):2363-70. https:// doi.org/10.1634/stemcells.2006-0686.

17. Schwartz MW, Seeley RJ, Zeltser LM, Drewnowski A, Ravussin E, Redman LM, et al. Obesity pathogenesis: an endocrine society scientific statement. Endocr Rev. 2017;38(4):267-96. https://doi.org/10.1210/er.2017-00111.

18. Liu GY, Liu J, Wang YL, Liu Y, Shao Y, Han Y, et al. Adipose-derived mesenchymal stem cells ameliorate lipid metabolic disturbance in mice. Stem Cells Transl Med. 2016;5(9):1162-70. https://doi.org/10.5966/sctm.20150239 .

19. Gummesson A, Nyman E, Knutsson M, Karpefors M. Effect of weight reduction on glycated haemoglobin in weight loss trials in patients with type 2 diabetes. Diabetes Obes Metab. 2017;19(9):1295-305. https://doi. org/10.1111/dom.12971.

20. Qi Y, Ma J, Li S, Liu W. Applicability of adipose-derived mesenchymal stem cells in treatment of patients with type 2 diabetes. Stem Cell Res Ther. 2019; 10(1):274. https://doi.org/10.1186/s13287-019-1362-2

21. Wu H, Ballantyne CM. Skeletal muscle inflammation and insulin resistance in obesity. J Clin Invest. 2017;127(1):43-54. https://doi.org/10.1172/JCl88880.

\section{Publisher's Note}

Springer Nature remains neutral with regard to jurisdictional claims in published maps and institutional affiliations. 